\title{
A Comparative Quantitative Proteomic Analysis of HCMV-Infected Cells Highlights pUL138 as a Multifunctional Protein
}

\author{
Yang Li ${ }^{1,2}\left(\mathbb{D}\right.$, Weijuan Shang ${ }^{3}$, Gengfu Xiao ${ }^{3}$, Lei-Ke Zhang ${ }^{3, *}$ and Congyi Zheng ${ }^{1,2, *}$ \\ 1 State Key Laboratory of Virology, College of Life Sciences, Wuhan University, Wuhan 430072, China; \\ 2012202040022@whu.edu.cn \\ 2 China Center for Type Culture Collection, Wuhan University, Wuhan 430072, China \\ 3 State Key Laboratory of Virology, Wuhan Institute of Virology, Chinese Academy of Sciences, \\ Wuhan 430071, China; shangweijuan@wh.iov.cn (W.S.); xiaogf@wh.iov.cn (G.X.) \\ * Correspondence: zhangleike@wh.iov.cn (L.-K.Z.); cctcc202@whu.edu.cn (C.Z.)
}

Received: 13 April 2020; Accepted: 21 May 2020; Published: 28 May 2020

\begin{abstract}
Human cytomegalovirus (HCMV) is a widespread virus that can establish life-long latent infection in large populations. The establishment of latent infection prevents HCMV from being cleared by host cells, and HCMV reactivation from latency can cause severe disease and death in people with immature or compromised immune systems. To establish persistent and latent infection in healthy individuals, HCMV encodes a large array of proteins that can modulate different components and pathways of host cells. It has been reported that pUL138 encoded by the UL133-UL138 polycistronic locus promotes latent infection in primary CD34+ hematopoietic progenitor cells (HPCs)

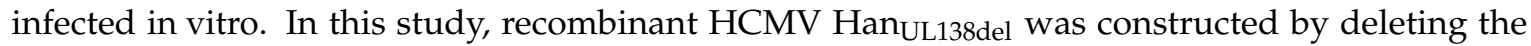
UL138 locus of Han, a clinical HCMV strain. Then, a comparative quantitative proteomic analysis of Han- and Han UL138del-infected MRC5 cells was performed to study the effect of pUL138 on host cells in the context of HCMV infection. Our results indicated that, during the early phase of HCMV infection, the innate immune response was differentially activated, while during the late phase of HCMV infection, multiple host proteins were differentially expressed between Han- and Han UL138del-infected cells, and these proteins are involved in the oxidation-reduction process, ER to Golgi vesicle-mediated transport, and extracellular matrix organization. Among these proteins, STEAP3, BORCS7, FAM172A, RELL1, and WDR48 were further demonstrated to affect HCMV infection. Our study provides a systematic view of the effect of pUL138 on the host cell proteome and highlights the proposition that multiple biological processes or host factors may be involved in the overall role of the UL133-UL138 polycistronic locus in HCMV persistence.
\end{abstract}

Keywords: HCMV; latent infection; UL138; quantitative proteomic

\section{Introduction}

Human cytomegalovirus (HCMV) belongs to the Betaherpesvirinae family and is a widespread virus that can establish lifelong latent infection in large populations. In the immunocompetent host, primary infection and reactivation of HCMV are typically asymptomatic [1]. However, in people with immature or compromised immune systems, opportunistic infection or reactivation can cause severe disease and death, particularly in solid-organ transplant patients [2]. Due to the complexity of HCMV-host interactions, the mechanisms by which HCMV regulates the establishment of latency or ensures successful reactivation from latency are currently not clear.

To establish persistent and latent infection in healthy individuals, HCMV encodes a large array of proteins that can modulate different components and pathways of host cells [3]. The genome of HCMV 
is a linear, double-stranded DNA of $\sim 230 \mathrm{~kb}$ in length. All clinical strains of HCMV contain a unique 13- to $15-\mathrm{kb}$ region of the genome named the ULb' region, which is lost upon serial passage of the virus in fibroblasts, resulting in viruses with higher replicative capacity but more restricted tropism [4-6]. Since 20 open reading frames encoded by the $\mathrm{ULb}^{\prime}$ region are nonessential for viral replication in human fibroblast cells, they have been hypothesized as being important for virus dissemination, latency, or pathogenesis in the human host [7].

The UL133-UL138 polycistronic locus contains genes within the ULb' region and is important for latency in the experimental CD34 ${ }^{+}$hematopoietic progenitor cell (HPC) model of latency [8-10]. Four novel proteins, namely pUL133, pUL135, pUL136, and pUL138, have been shown to be encoded by the UL133-UL138 locus [11], and it has been reported that pUL138 promotes a latent infection in primary CD34 ${ }^{+}$HPCs infected in vitro. The UL138 protein has been shown to increase cell surface levels of TNFR [12,13], although the significance of these surface alterations to viral infection is not completely understood.

HCMV infection can profoundly affect the infected cells, resulting in the modulation of cell metabolism, cell cycle, cell death and immune surveillance [14]. These fundamental changes to infected cells may contribute to the establishment of HCMV latency. To study the effect of HCMV infection on host cells, Weekes et al. performed a quantitative temporal proteomic analysis of HCMV-infected HFF cells, in which the modulation of intracellular signaling pathways by HCMV infection was deciphered [15]. Weekes et al. also performed quantitative proteomic analysis of THP-1 cells overexpressing pUL138 in order to monitor which host proteins were regulated by pUL138 and aiming to explore the intracellular signaling pathways that participate in the establishment of HCMV latency mediated by pUL138. They found that the expression of pUL138 resulted in a decrease in MRP1 and that loss of MRP1 and accumulation of the cytotoxic drug vincristine, an MRP1 substrate, decreased the replication of HCMV in latently infected $\mathrm{CD} 14^{+}$and $\mathrm{CD} 34^{+}$progenitors [16], suggesting that pUL138 may down regulate MRP1 expression to inhibit HCMV replication and thus help to establish persistent infection.

In this study, we constructed a recombinant HCMV strain based on the clinical strain Han, which was isolated from the urine sample of a Chinese infant with multiple developmental disorders [17].

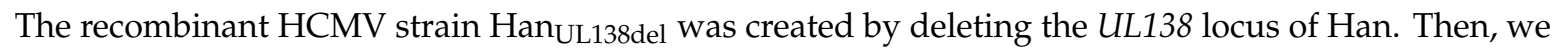
performed a comparative quantitative proteomic analysis of Han- and Han UL138del-infected MRC5 cells. Our results indicated that, during the early phase of HCMV infection, the innate immune response was differentially activated, while during the late phase of HCMV infection, multiple host proteins were differentially expressed between Han- and Han UL138del-infected cells. Our study provides a systematic view of the effect of pUL138 on the host cell proteome and highlights the proposition that multiple pUL138-regulating biological processes or host factors may contribute to the overall role of the UL133-UL138 polycistronic locus in HCMV persistence.

\section{Results}

\subsection{Construction of Recombinant HCMV Han ${ }_{U L 138 d e l}$}

To study the role of pUL138 in HCMV replication, we reconstituted two strains of HCMV, wild type HCMV and HCMV without pUL138, named Han and Han UL138del, respectively (Figure 1A). To monitor the growth kinetics of these two HCMV strains, MRC5 cells were infected with Han or Han UL138del at a multiplicity of infection (MOI) of 0.1 or 5. At different time intervals, the cells were collected, and the level of intracellular HCMV genome was measured by quantitative real-time PCR (RT-PCR). We found that when MRC5 cells were infected with HCMV at an MOI of 0.1, the relative level of intracellular HCMV DNA was lower in Han-infected cells (Figure 1B), while no significant changes were observed in MRC5 cells infected with Han or Han UL138del $_{\text {at }}$ an MOI of 5 (Figure 1C). We thus chose an MOI of five as the viral titer to perform quantitative proteomic analysis because the replication efficiencies of these two HCMV strains were similar under these conditions. 
A

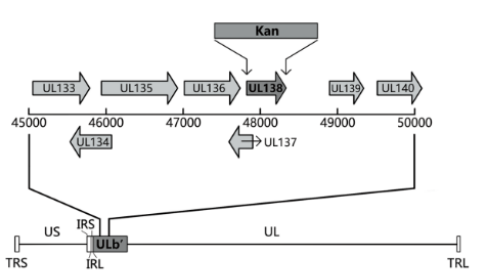

D

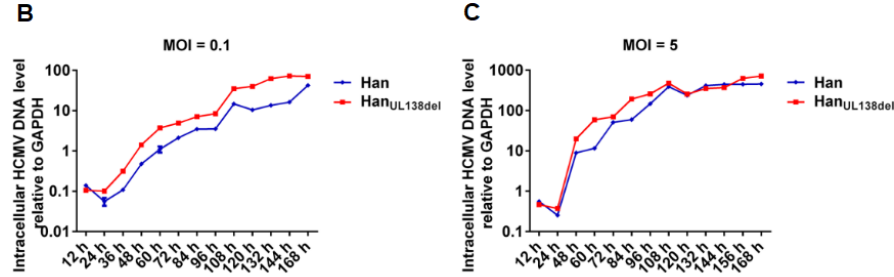

E

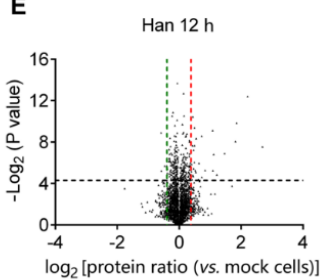

Han $96 \mathrm{~h}$

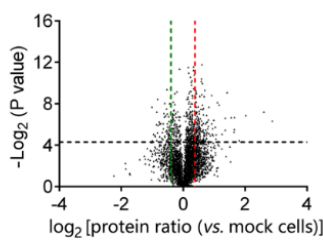

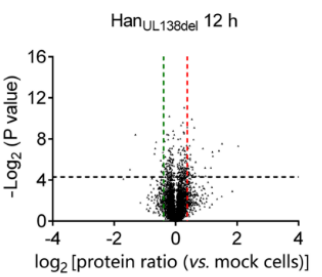

HanuL138del $96 \mathrm{~h}$

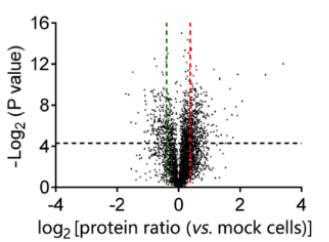

Figure 1. Comparative quantitative proteomic analysis of Han- and Han UL138del-infected MRC5 cells. (A) Construction of Han ${ }_{U L 138 d e l}-B A C$. The ORF of UL138 in the ULb' region was replaced by the kanamycin resistance cassette. (B) Replication kinetics curves of Han and Han UL138del in MRC5 cells infected at an MOI of 0.1 . Cells were harvested separately every $12 \mathrm{~h}$ for 7 days post infection. Intracellular levels of HCMV genomic DNA were detected with primers for UL44 by quantitative RT-PCR analysis. Three independent biological replicates were performed, and a representative experiment (with two technical replicates) from three biological replicates is shown. Results are shown as mean \pm SD. (C) Replication kinetics curves of Han and Han ${ }_{\text {UL138del }}$ in MRC5 cells infected at an MOI of 5. Three independent biological replicates were performed, and a representative experiment (with two technical replicates) from three biological replicates is shown. Results are shown as mean \pm SD. (D) Workflow for quantitative proteomic analysis. Cells were infected with Han or Han UL138del (at an MOI of 5) or mock-treated. At 12 h p.i. and 96 h p.i., cells were collected and lysed to extract proteins. Proteins in each sample were subjected to parallel denaturation and digestion. Digested samples were labeled with iTRAQ reagents and mixed together. The mixed peptides were fractionated and analyzed by LC-MS/MS analysis. (E) The ratio distribution of quantified proteins.

\subsection{Comparative Quantitative Proteomic Analysis of HCMV Han- and Han UL138del-Infected MRC5 Cells}

To perform quantitative proteomic analysis, MRC5 cells were infected with HCMV Han (MOI of 5) or Han UL138del (MOI of 5) or mock-treated, and the cells were collected at 12 and $96 \mathrm{~h}$ post infection (h p.i.) (Figure 1D). Proteins were then extracted and subjected to trypsin digestion, and the digested peptides were labeled with iTRAQ reagents according to the manufacturer's instructions. Then, equal amounts of the labeled peptides were mixed and subjected to SCX fractionation and LC-MS/MS analysis. Protein identification was performed with ProteinPilot. Three independent biological replicates were performed.

A total of 5119 host proteins were quantified. At 12 h p.i., 48 were upregulated and 29 were downregulated in Han-infected cells, while 79 were upregulated and 58 were downregulated in

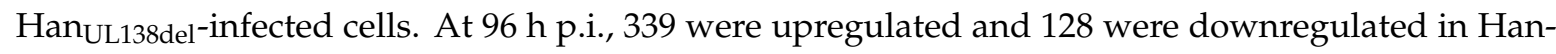
infected cells, while 494 were upregulated and 221 were downregulated in Han UL138del-infected cells (Figure 1E, Supplementary Table S1-S3).

To validate our MS data, both quantitative RT-PCR and Western blot analysis were performed. Western blot analysis was performed on PML, PSMB8, HNRNPD, RELL1 and SPARC, an exemplary set of proteins, and CANX was selected as the loading control [15]. The intensity of the band 
was normalized to that in mock-treated cells, and relative fold change were calculated (Figure 2A). We found that, although the fold changes were not identical between the Western blot and MS results, the tendencies were the same. For example, both MS data and WB analysis indicated that PML was upregulated at both time points, while PSMB8 was only upregulated at $96 \mathrm{~h}$ p.i. in both HCMV

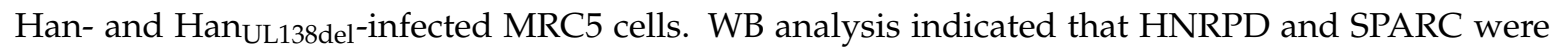
downregulated at $96 \mathrm{~h}$ p.i., while SPARC was slightly downregulated at $12 \mathrm{~h}$ p.i. in both HCMV Hanand Han $U L 138 \mathrm{del}$-infected MRC5 cells, and this result was consistent with the MS results.
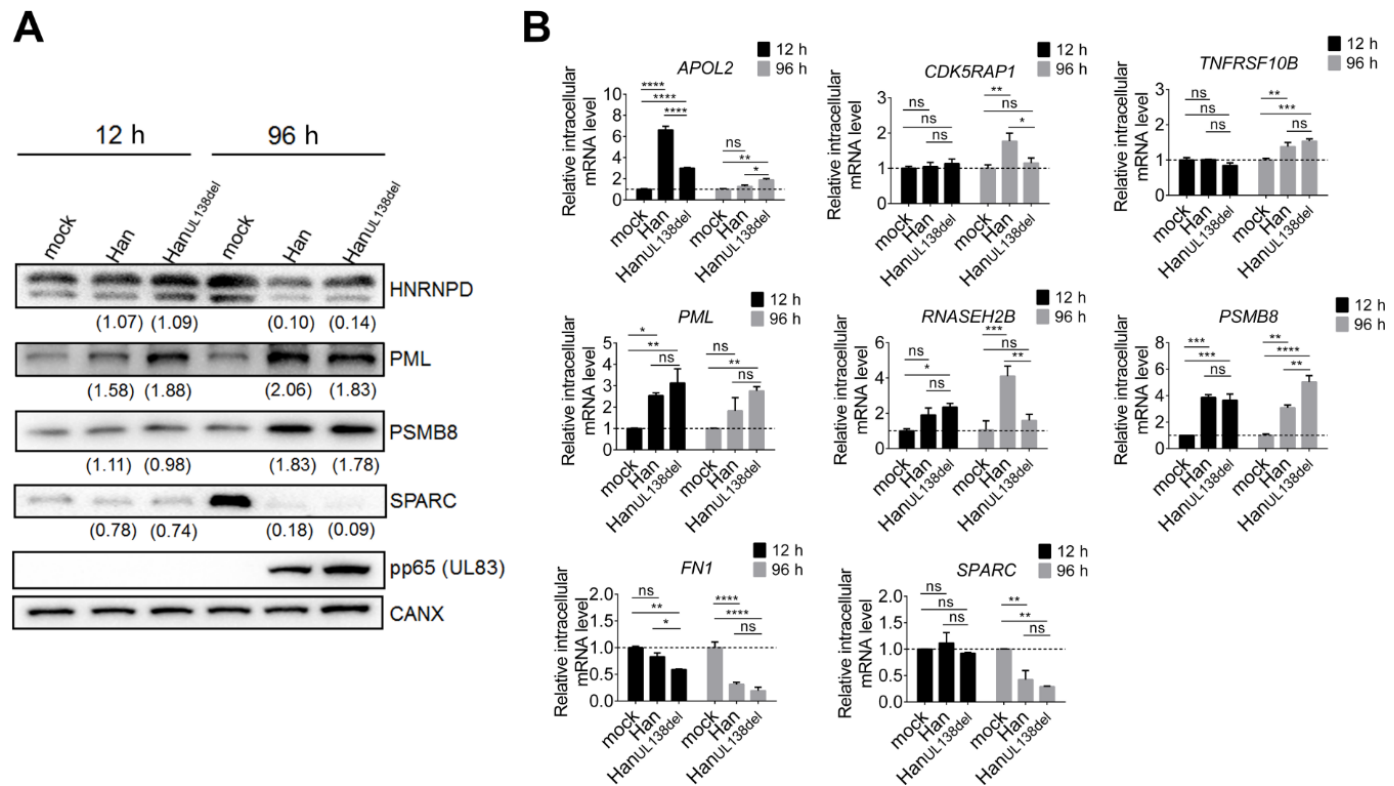

Figure 2. Validation of MS results. (A) Western blot analysis of MRC5 cells infected with Han or Han $_{\mathrm{UL} 138 \mathrm{del}}$ at an MOI of 5 . MRC5 cells were harvested at $12 \mathrm{~h}$ p.i. or $96 \mathrm{~h}$ p.i., and subjected to Western blot analysis. The intensity of each band was quantitated with Quantity One software and normalized to mock-treated cell band intensity. The numbers with brackets marked below the band represent the relative fold changes. (B) Quantitative real-time PCR analysis of selected proteins in cells (MRC5) infected with Han, HanUL138del (at an MOI of 5) or mock-treated. MRC5 cells were harvested at $12 \mathrm{~h}$ p.i. or $96 \mathrm{~h}$ p.i. and intracellular RNAs were extracted and reverse transcribed to cDNA. The relative mRNA levels of selected proteins were measured by quantitative RT-PCR analysis. Three replicates were performed, and results are shown as mean \pm SD. ${ }^{*} p<0.05 ;{ }^{* *} p<0.01 ;{ }^{* * *} p<0.001 ;{ }^{* * * *} p<0.0001$; ns, no significance.

To further validate our MS results, we also performed quantitative RT-PCR analysis on selected differentially regulated proteins. We found that the mRNA levels of APOL2, CDK5RAP1, TNFRSF10B, PML, RNASEH2B, and PSMB8 were upregulated, while the mRNA levels of FN1 and SPARC were downregulated by infection with HCMV Han and/or Han UL138del (Figure 2B), and these tendencies were consistent with the MS results, suggesting that these proteins were regulated at the mRNA level. Both WB and quantitative RT-PCR analysis supported our MS data.

\subsection{The Innate Immune Response Was Differentially Regulated between Han and Han UL138del $_{1}$}

At $12 \mathrm{~h}$ p.i., 170 proteins were differentially regulated by Han and/or Han UL138del, and we then performed gene ontology (GO) analysis on these proteins. We found that the proteins could be grouped into multiple biological processes, including proteins involved in the innate immune response, ubiquitin system, and vesicular transport (Figure 3). No biological processes were specifically regulated by either

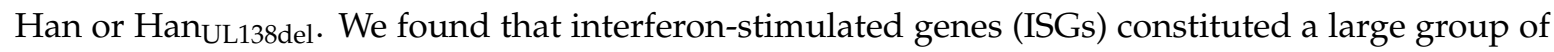
upregulated proteins. Moreover, the upregulation of ISGs by HCMV has also been observed in previous studies $[15,18]$. However, compared to Han-infected cells, we found that the fold changes of multiple 


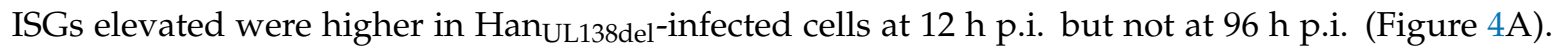
To confirm this result, we performed WB analysis of SAMD9, a reported ISG with antiviral activity [19]. As shown in Figure 4B, the intracellular protein level of SAMD9 was higher in Han-infected cells than in Han UL138del-infected cells at $12 \mathrm{~h}$ p.i. (Figure 4B). We also performed quantitative RT-PCR analysis on selected ISGs and found that the mRNA levels of SAMD9, MDA5, ISG15, ISG56, and STAT1 were

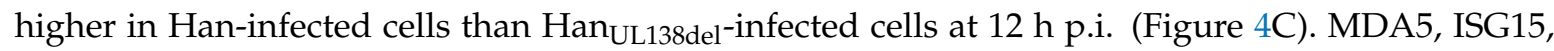
and ISG56 have been reported that can be upregulated by HCMV infection [15,20], while the HCMV immediate early gene 1 (IE1) can activate STAT1 by re-routing IL-6 signaling [21]..The above results indicated that at the early stage post HCMV infection, both HCMV strains activated the expression of ISGs, while in cells infected with Han, which can express pUL138, a more significant increase in ISG production was observed.

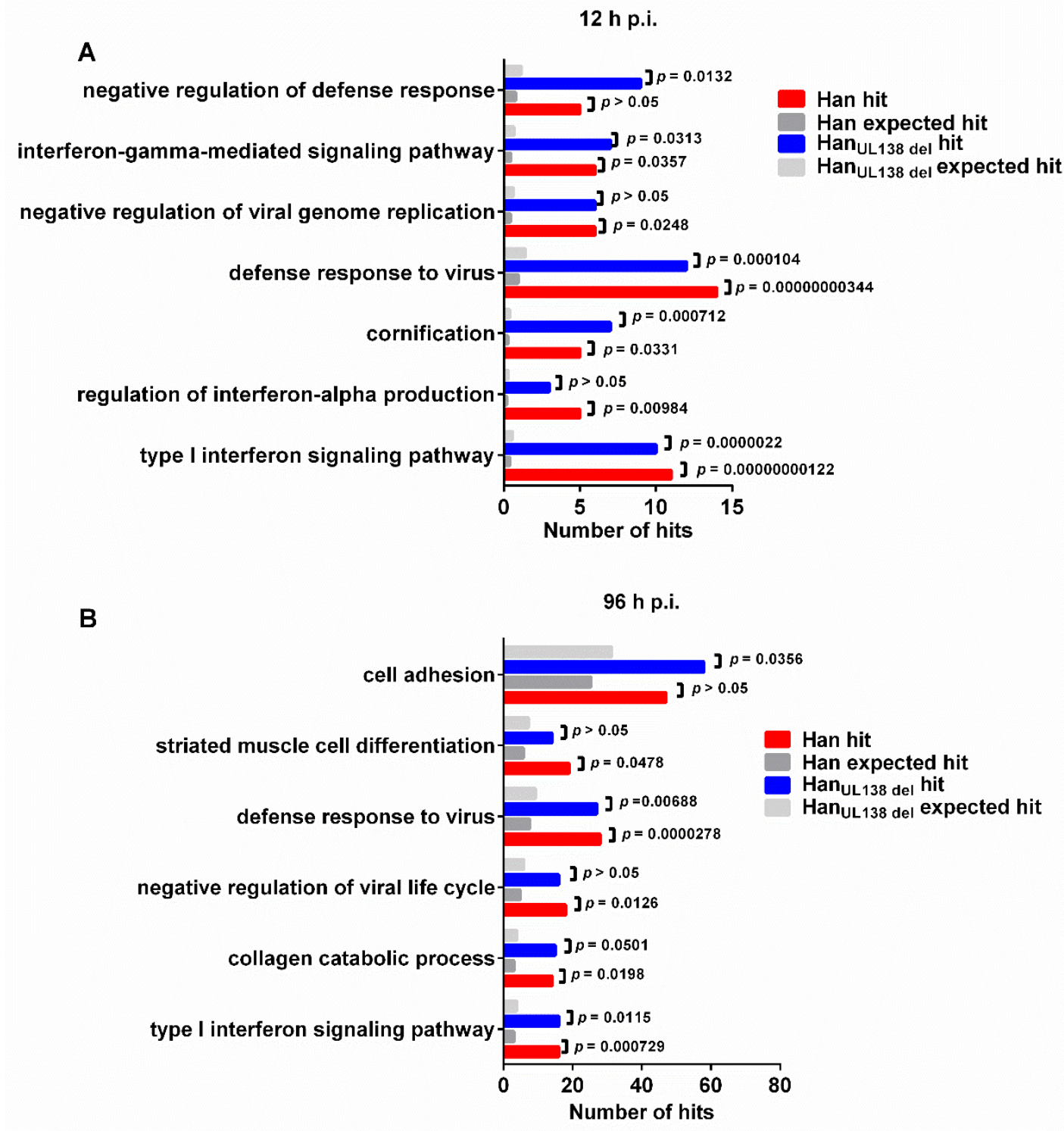

Figure 3. Gene Ontology analysis (biological process) of differentially regulated proteins at $12 \mathrm{~h}$ p.i. or 96 h p.i.. Differentially regulated proteins at $12 \mathrm{~h}$ p.i. (A) or $96 \mathrm{~h}$ p.i. (B) were subjected to DAVID v6.8. The regulated proteins were grouped based on their roles in biological processes, and a statistical overrepresentation test was performed to determine which biological process was overrepresented by differentially regulated proteins. Only the overrepresented categories are presented here. Expected hits indicate the presumed number of hits in this category. 
A
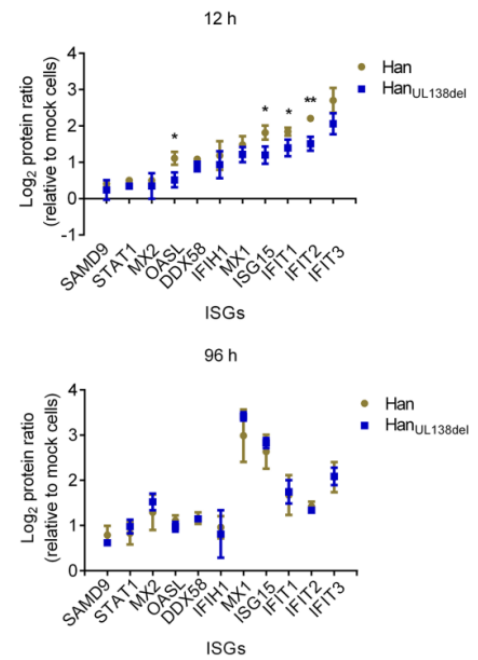

B

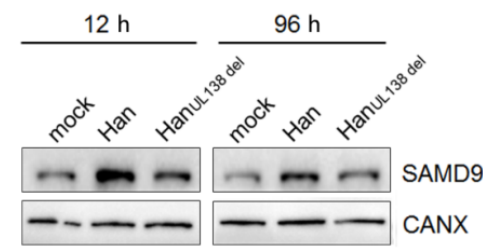

C
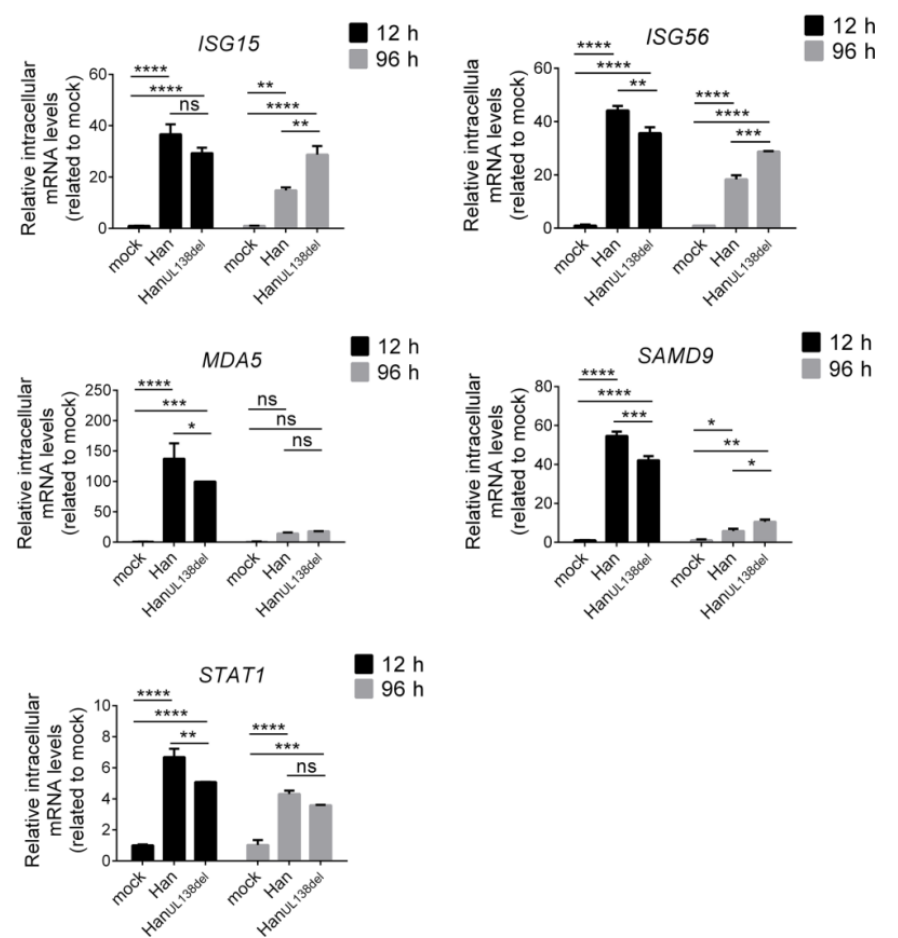

Figure 4. Differential regulation of the innate immune response by Han and Han UL138del at early stage post infection. (A) Differential expression of ISGs in Han- and Han UL138del-infected MRC5 cells at $12 \mathrm{~h}$ p.i. or $96 \mathrm{~h}$ p.i.. Y-axis: protein ratios quantified by mass spectrometry (relative to mock-treated cells). (B) Western blot analysis of SAMD9 levels in Han- and Han UL138del-infected MRC5 cells at $12 \mathrm{~h}$ p.i. or $96 \mathrm{~h}$ p.i.. SAMD9 was selected as a representative ISG. (C) Quantitative RT-PCR analysis of selected

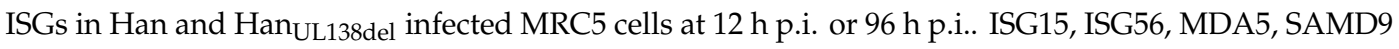
and STAT1 were selected as representative ISGs. H: h p.i.. Three replicates were performed, and results are shown as mean $\pm \mathrm{SD} .{ }^{*} p<0.05 ;{ }^{* *} p<0.01 ;{ }^{* * *} p<0.001 ;{ }^{* * *} p<0.0001$; ns, no significance.

\subsection{Proteins Differentially Regulated by HCMV Han and Han ${ }_{U L 138 d e l}$ at 96 h p.i. Can Affect HCMV Replication}

Among the 990 host proteins regulated at $96 \mathrm{~h}$ p.i., we found that multiple proteins were differentially regulated between HCMV Han and HCMV Han UL138del. Next, we explored whether these proteins could affect the replication of HCMV. Briefly, MRC5 cells were transfected with specific siRNAs, and the knockdown efficiency was examined with quantitative RT-PCR (Supplementary Figure S2). Forty-eight hours post siRNA transfection, MRC5 cells were infected with HCMV Han or

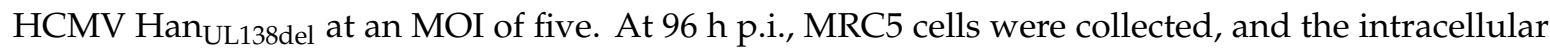
level of HCMV DNA was measured by quantitative RT-PCR. As shown in Figure 5A, knocking down BORCS7, FAM172A, RELL1, and WDR48 reduced intracellular HCMV DNA levels, while knocking down STEAP3 increased intracellular HCMV DNA levels. We also determined the effect of knocking down target proteins on the production of HCMV virions. At $48 \mathrm{~h}$ post transfection, MRC5 cells were infected with HCMV Han or HCMV Han collected, and the viral titer was measured. Knocking down BORCS7, FAM172A, RELL1, and WDR48 reduced the production of HCMV virions, while knocking down STEAP3 promoted the production of HCMV virions (Figure 5B). The above results indicated that, at $96 \mathrm{~h}$ p.i., multiple host proteins were differentially regulated between Han and Han $\mathrm{UL}_{138 \mathrm{del}}$ in infected MRC5 cells, and among these proteins, BORCS7, FAM172A, RELL1, and WDR48 can promote HCMV infection, while STEAP3 can inhibit HCMV replication. 
A
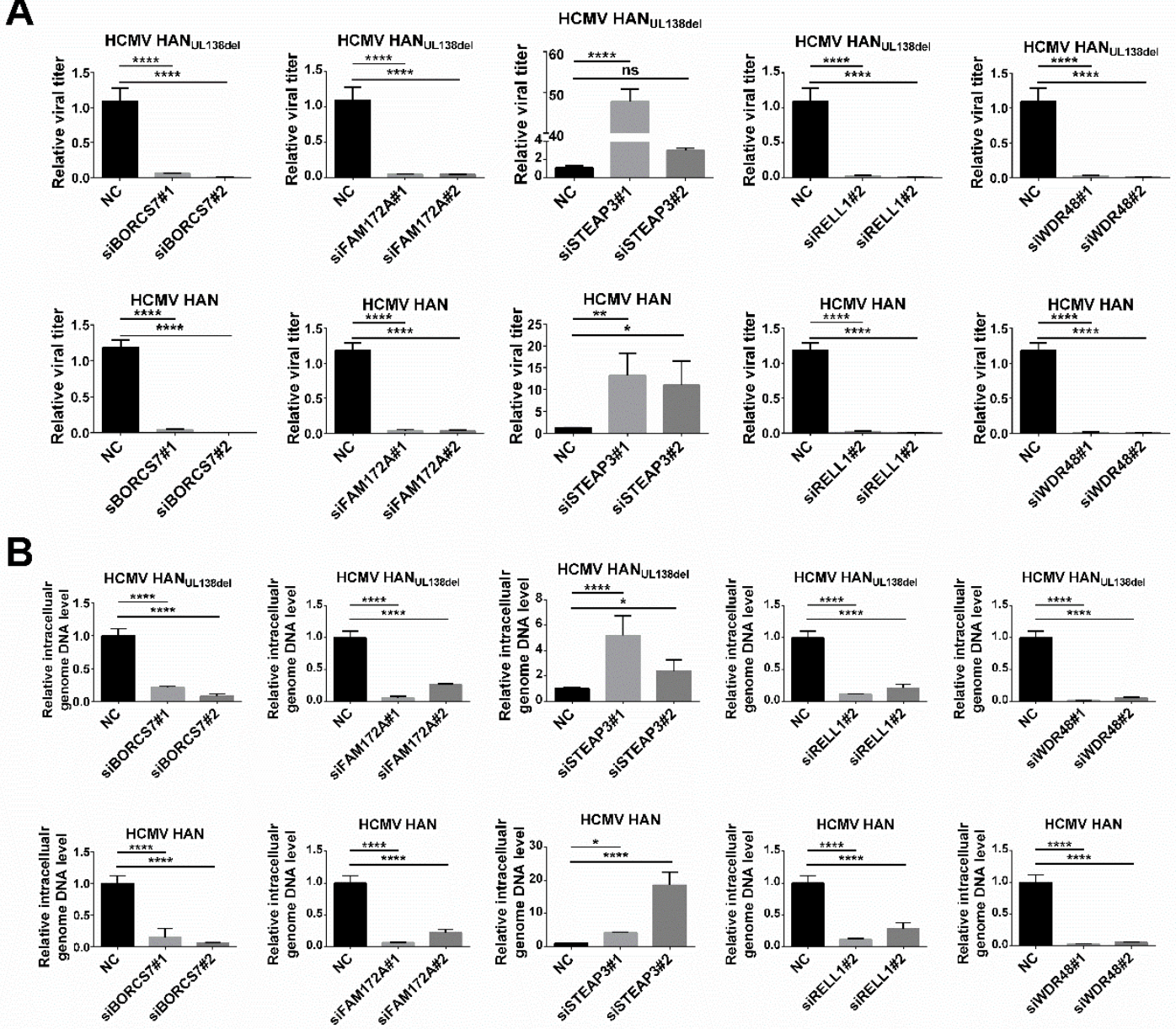

Figure 5. Host proteins differentially regulated by pUL138 at the late stage post HCMV infection can affect HCMV replication. (A) The effect of knockdown of BORCS7, FAM172A, RELL1, WDR48 or STEAP3 on HCMV production. MRC5 cells were transfected with siRNA or NC. At $48 \mathrm{~h}$ post transfection, the cells were infected with Han or Han UL138del at an MOI of 0.1 or mock-treated. At $5 \mathrm{~d}$ post infection, cell culture supernatants were collected, and viral titers in the supernatants were measured. (B) The effect of knockdown of BORCS7, FAM172A, RELL1, WDR48 or STEAP3 on intracellular HCMV DNA levels. MRC5 cells were transfected with siRNA or NC. At $48 \mathrm{~h}$ post transfection, the cells were infected with Han or Han UL138del at an MOI of 0.1 or mock-treated. At $5 \mathrm{~d}$ post infection, the cells were harvested to extract DNA. Intracellular levels of HCMV genomic DNA were detected with primers for UL44 by quantitative RT-PCR analysis.Three replicates were performed, and results are shown as mean $\pm \mathrm{SD} .{ }^{*} p<0.05 ;{ }^{* *} p<0.01 ;{ }^{* * *} p<0.001 ;{ }^{* * * *} p<0.0001$; ns, no significance.

\section{Discussion}

It has been reported that pUL138 plays an important role in the establishment of latent HCMV infection. To address how pUL138 affects host cell surface protein expression during latent HCMV infection, Weekes et al. performed a quantitative proteomic analysis on myeloid cells with and without pUL138 [16] and found that several differentially regulated proteins may participate in the establishment of latent HCMV infection. However, the effect of pUL138 on the whole cell proteome in the context of HCMV infection is still unknown. To this end, we constructed an HCMV strain without UL138 (HCMV Han UL138del), derived from the HCMV clinical strain Han, and performed comparative quantitative proteomic analysis of MRC5 cells infected with HCMV Han and HCMV Han UL138del. A total of 5119 proteins were quantified at both 12 and $96 \mathrm{~h}$ p.i., and hundreds of differentially expressed proteins were identified (Supplementary Table S1-S3 and Figure S1). Considering that quantification 
with mixed protein sources from host and virus might cause errors, we thus employed Western blot and quantitative RT-PCR analysis on selected proteins to verify our MS results. GO analysis indicated that, at $12 \mathrm{~h}$ p.i., the early stage post HCMV infection, proteins involved in innate immune response, ubiquitin system, and vesicular transport were differentially regulated by both HCMV Han and HCMV Han UL138del infection. Further examination of protein ratio distributions indicated that the levels of regulated ISGs were higher in HCMV Han-infected MRC5 cells than in HCMV Han UL138del-infected MRC5 cells, suggesting that the production of ISGs was more highly activated after HCMV Han infection. It has been reported that pUL138 can be expressed at $6 \mathrm{~h}$ p.i. in HCMV-infected cells [22], and we speculate that the expression of pUL138 at this time point might activate the production of ISGs and subsequently suppress HCMV replication to help establish latent infection. Indeed, we found that HCMV replication was lower in the presence of pUL138 when the cells were infected with a low MOI of HCMV (Figure 1B).

As HCMV started to replicate and more viral proteins were expressed, a more significant effect on the host proteome was observed in HCMV infected cells at $96 \mathrm{~h}$ p.i., the late stage post HCMV infection. However, the expression levels of ISGs were comparable between Han- and Han UL138del-infected cells at this time point, which may be because other viral proteins that have been reported to affect the innate immune response were also expressed. More differentially regulated proteins were identified at $96 \mathrm{~h}$ p.i., and we found that multiple proteins were differentially regulated between Han- and Han UL138del-infected cells. We then performed functional analysis of selected proteins and found that BORCS7, FAM172A, STEAP, RELL1, and WDR48 could affect HCMV replication.

Among these proteins, RELL1 is a membrane protein that can induce cellular death in HEK 293 epithelial cells [23]. Here, we found that intracellular level of RELL1 in Han-infected cells was

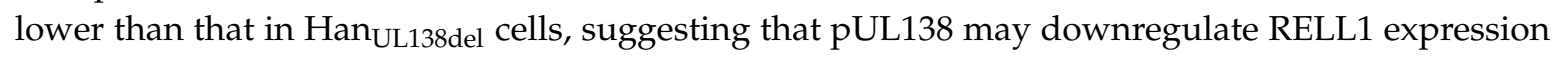
during HCMV infection. A previous quantitative proteomic analysis also indicated that RELL1 was downregulated in pUL138-expressing THP-1 cells [16]. Thereafter, we explored whether RELL1 could affect HCMV replication and found that knocking down RELL1 could decrease both HCMV replication and the virus titer (Figure 5), suggesting that RELL1 can promote HCMV replication. The downregulation of RELL1 by pUL138 during HCMV infection may inhibit HCMV replication, thus favoring the establishment of latent infection.

WDR48 is a regulator of deubiquitinating complexes [24]. It has been reported that herpes virus saimiri Tip protein can interact with WDR48 to down regulate $\mathrm{T}$ cell receptor (TCR) and CD4 surface expression. Moreover, herpes viruses may employ this mechanism to deregulate lymphocyte receptor expression to disarm host immune control [25]. Here, we found that WDR48 was

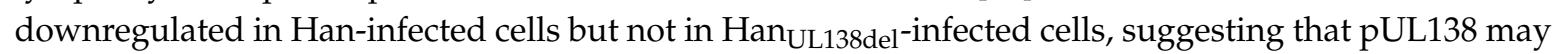
decrease intracellular WDR48 levels. We further found that knocking down WDR48 impaired HCMV replication, suggesting that pUL138 may downregulate this protein to inhibit HCMV replication. We also found that the protein level of FAM172A in HCMV Han-infected cells was lower than that in

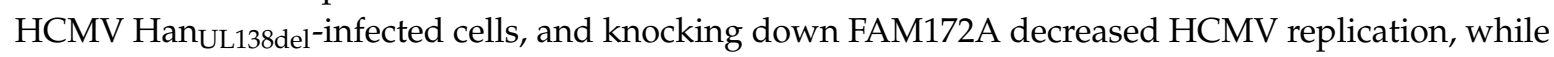
the intracellular protein level of STEAP3 in HCMV Han-infected cells was higher than that in HCMV Han $_{U L 138 d e l}$-infected cells, and knocking down STEAP3 increased HCMV replication, suggesting that pUL138 may upregulate STEAP3 to inhibit HCMV replication.

In conclusion, our study provides a comprehensive view of the effects of pUL138 on the host cell proteome in the context of HCMV infection. Our results indicated that, during the early phase of HCMV infection, the innate immune response was differentially activated as a result of pUL138 expression. During the late phase of HCMV infection, STEAP3, BORCS7, FAM172A, RELL1 and WDR48 were differentially expressed between Han- and Han $\mathrm{UL}_{138 \mathrm{del}}$-infected cells, and further functional studies indicated that all of these proteins could affect HCMV infection. Our study provides a systematic view of the effect of pUL138 on the host cell proteome and highlights the proposition that multiple biological processes or host factors may contribute to the overall role of the UL133-UL138 polycistronic locus in HCMV persistence. 


\section{Materials and Methods}

\subsection{Cells}

Human fetal lung fibroblasts (MRC5), HEK 293T cells and HeLa cells were obtained from CCTCC. MRC5 cells were cultured in minimum Eagle's medium (MEM) supplemented with 10\% fetal bovine serum (FBS), $100 \mathrm{U} / \mathrm{mL}$ penicillin, and $100 \mu \mathrm{g} / \mathrm{mL}$ streptomycin at $37^{\circ} \mathrm{C}$ with $5 \% \mathrm{CO}_{2}$. HEK293T and HeLa cells were cultured in Dulbecco's modified Eagle's medium (DMEM) supplemented with $10 \%$ FBS, $100 \mathrm{U} / \mathrm{mL}$ penicillin, and $100 \mu \mathrm{g} / \mathrm{mL}$ streptomycin at $37^{\circ} \mathrm{C}$ with $5 \% \mathrm{CO}_{2}$.

\subsection{Viruses}

HCMV Han DNA was a gift from Dr. Minhua Luo (Wuhan Institute of Virology, CAS) [17]. HCMV Han was cloned into a bacterial artificial chromosome (BAC) that contains green fluorescent protein (GFP) to obtain Han-BAC DNA. Han-BAC DNA was amplified in recombinogenic Escherichia coli strain EL350. Briefly, Han BAC DNA was electroporated into E. coli EL350 using a Gene Pulser Xcell (Bio-Rad, Hercules, CA, USA) with $0.1 \mathrm{~cm}$-gap cuvette at $1600 \mathrm{~V}$. An EL350 clone containing Han BAC was selected on LB agar plates supplemented with $12.5 \mu \mathrm{g} / \mathrm{mL}$ chloramphenicol and then amplified in LB liquid medium supplemented with $12.5 \mu \mathrm{g} / \mathrm{mL}$ chloramphenicol. Then, EL350 were collected, and Han-BAC DNA was purified with NucleoBond Xtra Midi (Macherey-Nagel, Düren, Germany).

To obtain Han-BAC DNA without UL138, a DNA segment carrying a kanamycin resistance cassette flanked by sequences homologous to sequences flanking UL138 in the Han genome was amplified from the pGBKT7 plasmid and electroporated into E. coli EL350, in which UL138 was replaced by kan via

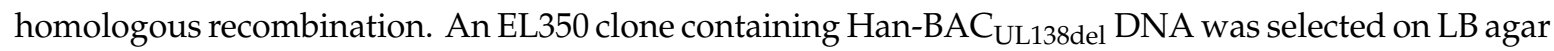
plates supplemented with $12.5 \mu \mathrm{g} / \mathrm{mL}$ chloramphenicol and $30 \mu \mathrm{g} / \mathrm{mL}$ kanamycin. Han-BAC UL138del DNA was extracted as described above.

To reconstitute the viruses, BAC DNA and the pcDNA3.1-UL82 plasmid were electroporated into MRC5 cells with a 0.4-cm-gap cuvette at $260 \mathrm{~V}$. HCMV virions were collected after MRC5 cells were subjected to three freeze-thaw cycles. Cell debris was removed from the suspension by centrifugation at $2000 \mathrm{rpm}$ for $10 \mathrm{~min}$ at $4{ }^{\circ} \mathrm{C}$. Virions were concentrated by ultracentrifugation through a $35 \%$ (wt/vol) sucrose cushion at 25,000 rpm $(\sim 77,000 \times \mathrm{g})$ in an SW32 rotor (Beckman Coulter, USA) for $2 \mathrm{~h}$ at $4{ }^{\circ} \mathrm{C}$. Virus particles were resuspended in MEM with 10\% FBS and 1\% DMSO and stored at $-80{ }^{\circ} \mathrm{C}$.

\subsubsection{Viral DNA Purification}

MRC5 cells were seeded onto 12 -well plates at $1 \times 10^{5}$ cells per well and infected with HCMV Han or Han UL138del at an MOI of 0.1 or 5 and were harvested using cell scarpers at different time intervals and stored at $-80^{\circ} \mathrm{C}$. All samples were lysed with Buffer A in the TIANamp Genomic DNA Kit (Tiangen, Beijing, China), and DNA was purified following the manufacturer's protocol.

\subsubsection{RNA Purification}

Cells were lysed in RNAiso Plus (Takara, Dalian, China) to extract total RNA. Then, RNA was reverse transcribed to $\mathrm{cDNA}$ using $\mathrm{SYBR}^{\circledR}$ Premix EX Taq ${ }^{\mathrm{TM}}$ II (Takara, Dalian, China) following the manufacturer's protocol. The relative mRNA levels of selected proteins were measured by quantitative RT-PCR analysis.

\subsubsection{Quantitative Real-Time PCR}

Quantitative RT-PCR was performed in a total volume of $20 \mu$ using TB Green Premix Ex Taq ${ }^{\mathrm{TM}}$ II (Takara, Dalian, China) on an ABI StepOnePlus ${ }^{\mathrm{TM}}$ Real-Time PCR System (Applied Biosystems, Foster City, CA) using the following protocol: $50{ }^{\circ} \mathrm{C}$ for $2 \mathrm{~min}$ and $95^{\circ} \mathrm{C}$ for $2 \mathrm{~min}$, then 40 cycles of $95{ }^{\circ} \mathrm{C}$ for $15 \mathrm{~s}$ and $60^{\circ} \mathrm{C}$ for $1 \mathrm{~min}$, ending with the melting curve stage. 


\subsubsection{Protein Extraction}

HCMV-infected or mock-treated MRC5 cells were collected and washed with prechilled PBS three times. Cell pellets were resuspended in lysis buffer ( $8 \mathrm{M}$ urea/0.2 $\mathrm{M}$ Tris, $\mathrm{pH} 8.0)$ and subjected to sonication. After centrifugation, supernatants containing the extracted proteins were collected, and protein abundance was measured with a bicinchoninic acid (BCA) assay. Extracted proteins were reduced with $10 \mathrm{mM}$ DTT at $56{ }^{\circ} \mathrm{C}$ for $30 \mathrm{~min}$, alkylated with $40 \mathrm{mM}$ iodoacetamide in the dark for $30 \mathrm{~min}$, and left in light at room temperature for $2 \mathrm{~h}$. Proteins were digested with trypsin (Promega) at a ratio of 1:50 (trypsin/protein $\mathrm{w} / \mathrm{w}$ ) overnight at $37^{\circ} \mathrm{C}$, and the digested peptides were desalted with SepPak C18 cartridge (Waters) and dried by Speed Vac (Thermo).

\subsection{5. iTRAQ Labeling and LC-MS/MS Analysis}

For iTRAQ labeling, $100 \mu \mathrm{g}$ of peptides from HCMV Han-, HCMV HanUL138del- or mock-infected cells were labeled with different iTRAQ reagents according to the manufacturer's instructions (SCIEX), and equal amounts of labeled peptides were mixed and desalted. The mixed peptides were fractionated into 8 fractions using strong cation exchange (SCX) as previously described [26]. The fractionated peptides were dried by Speed Vac and stored at $-80^{\circ} \mathrm{C}$.

LC-MS/MS analysis using NanoLC-Ultra 1D plus (Eksigent) was performed on a quadrupole-TOF LC/MS/MS mass spectrometer (TripleTOF 5600+, SCIEX) equipped with a nanospray source. Peptides dissolved in loading buffer $\left(2 \% \mathrm{ACN} / 97.9 \% \mathrm{H}_{2} \mathrm{O} / 0.1 \%\right.$ formic acid) were first loaded onto a $\mathrm{C} 18$ trap column ( $5 \mu \mathrm{m}, 5 \times 0.3 \mathrm{~mm}$, Agilent Technologies) and then eluted into a C18 analytical column $(75 \mu \mathrm{m}$ $\times 150 \mathrm{~mm}, 3 \mu \mathrm{m}$ particle size, $100 \AA \AA$ A pore size, Eksigent). Mobile phase A (3\% DMSO, 96.9\% H2O, 0.1\% formic acid) and mobile phase $\mathrm{B}(3 \% \mathrm{DMSO}, 96.9 \% \mathrm{ACN}, 0.1 \%$ formic acid) were used to establish a 100 min gradient, which comprised of: $0 \mathrm{~min}$ in $5 \%$ B, $65 \mathrm{~min}$ of $5-23 \% \mathrm{~B}, 20 \mathrm{~min}$ of $23-52 \% \mathrm{~B}, 1 \mathrm{~min}$ of $52-80 \% \mathrm{~B}$, the gradient was maintained in $80 \% \mathrm{~B}$ for $4 \mathrm{~min}$, followed by $0.1 \mathrm{~min}$ of $80-85 \% \mathrm{~B}$, and a final step in $5 \%$ B for $10 \mathrm{~min}$. The constant flow rate was set at $300 \mathrm{~nL} / \mathrm{min}$. For MS/MS analysis, each scan cycle consisted of one full-scan mass spectrum (with $\mathrm{m} / \mathrm{z}$ ranging from 350 to 1500 and charge states from 2 to 5) followed by $20 \mathrm{MS} / \mathrm{MS}$ events. The threshold count was set to 120 to activate MS/MS accumulation, and former target ion exclusion was set for $18 \mathrm{~s}$. Mass spectra were extracted by Peakview v2.0 (SCIEX).

\subsection{Experimental Design and Statistical Rationale}

Three independent biological replicates were performed, and peptides from three biological replicates were analyzed by LC-MS/MS independently. The mass spectrometry proteomics data have been deposited to the ProteomeXchange Consortium [27] via the PRIDE partner repository with the dataset identifier $<$ PXD015931 $>$.

MS spectra were subjected to ProteinPilot v5.0.1 (SCIEX) analysis for peptide identification and quantification. A concatenated database $(\mathrm{n}=20355)$ containing the HCMV protein sequence and the UniProt_Human database (2016/05) was used. Search parameters were as follows: sample type: iTRAQ 8plex (peptide labeled); cysteine alkylation: iodoacetamide; digestion: trypsin; miss cleavage tolerance: 2; fixed modification: carbamidomethyl Cys; variable modification: none; MS1 initial mass error tolerance value: 0.05 Dalton; MS2 initial mass error tolerance value: 0.1 Dalton; Instrument: TripleTOF 5600. The false discovery rate (FDR) analysis in ProteinPilot uses the "decoy database searching" strategy, and in this study the FDRs of the ProteinPilot search results were all set as lower than $1 \%$ at the protein level.

For protein quantification, all quantified peptides were exported, and only the peptides with a confidence score $>95 \%$ were kept for further analysis. In each replicate, the protein ratio was calculated by weighted average ratios of its peptides, with peptide intensity as the weight. The protein ratio values used for bioinformatics analysis were the weighted averages of the three biological replicates, while the $p$ value for the protein ratio was calculated and further corrected with multiple Bonferroni 
correction (Supplementary Table S1). All quantified proteins presented had at least two quantified peptides. The cutoff for differentially regulated proteins was set as described in a previous study $[28,29]$. Briefly, the Gaussian distribution of protein ratios was analyzed, and values deviating from the mean of the normally distributed data by 3.3 standard deviations were considered as cutoff values. Only proteins that met the following two criteria were considered differentially regulated: (1) with ratios $>$ upregulated or $<$ downregulated cutoff values, and (2) with corrected protein ratio $p$ value $<0.05$.

\subsection{Gene Ontology (GO) Analysis}

To perform GO analysis, differentially regulated proteins were submitted to DAVID 6.8 [30]. Proteins were classified into different categories, and a statistical over-representation test was performed. $p$ values were assessed with a binomial test and corrected for multiple testing using a Bonferroni procedure. Only categories with a $p$ value $<0.05$ were considered as over- or under- represented.

\subsection{Western Blotting}

Cells were lysed in RIPA lysis buffer (Beyotime, Shanghai, China) or 1X SDS-loading buffer with PMSF. Protein lysates were fractionated by sodium dodecyl sulfate-polyacrylamide gel electrophoresis in 10 to $15 \%$ gels and transferred to $0.2 \mu \mathrm{m}$ PVDF membranes in semi-dry transfer system (Bio-Rad, Hercules, CA, USA). Membranes were blocked in Tris-buffered saline with 0.1\% Tween-20 (TBST) containing 5\% nonfat dry milk for $2 \mathrm{~h}$ at room temperature. Membranes were incubated with primary antibodies in TBST overnight at $4{ }^{\circ} \mathrm{C}$. After washing with TBST (three times, 20 min each), membranes were incubated with horseradish peroxidase-labeled secondary antibodies in TBST containing 5\% nonfat dry milk for $1 \mathrm{~h}$ at room temperature. The bands were developed with enhanced chemiluminescence (ECL) (Millipore, Billerica, MA) and visualized by the ChemiDoc MP Imaging System (Bio-Rad, Hercules, CA, USA). The following antibodies (Abs) were used: anti-HNRNPD polyclonal Ab (ProteinTech, Wuhan, China), anti-PML polyclonal Ab (ProteinTech, Wuhan, China), anti-PSMB8 polyclonal Ab, anti-SPARC polyclonal Ab, anti-pp65 monoclonal Ab (Santa Cruz Biotechnology, Dallas, $\mathrm{TX}$ ), and anti-CANX polyclonal Ab (ABclonal, Wuhan, China).

Supplementary Materials: The following Supplementary Materials can be found online: Table S1. Ratio of protein quantified in HCMV infected cells; Table S2. Protein ratios of Han vs. HanUL138del at 12 and $96 \mathrm{~h}$ p.i.; Table S3. Protein ratios of late infection (96 h p.i.) vs. early infection (12 h p.i.) in Han and HanUL138del infected cells; Figure S1. The ratio distribution of quantified proteins; Figure S2. Knockdown efficiency of target gene-specific siRNAs.

Author Contributions: Conceptualization, L.-K.Z. and C.Z.; Data curation, Y.L.; Funding acquisition, L.-K.Z.; Investigation, Y.L. and W.S.; Project administration, G.X. and C.Z.; Supervision, G.X., L.-K.Z. and C.Z.; Validation, Y.L.; Writing-original draft, Y.L.; Writing-review \& editing, L.-K.Z. All authors have read and agreed to the published version of the manuscript.

Funding: This work was supported by the National Science and Technology Major Project (No. 2018ZX10101004), the National Natural Science Foundation of China (no. 31500144).

Acknowledgments: We thank the Core Facility and Technical Support Facility of the Wuhan Institute of Virology for their technical assistance.

Conflicts of Interest: The authors declare no conflict of interest. The founding sponsors had no role in the design of the study; in the collection, analyses, or interpretation of data; in the writing of the manuscript, and in the decision to publish the results.

\section{References}

1. Rossini, G.; Cerboni, C.; Santoni, A.; Landini, M.P.; Landolfo, S.; Gatti, D.; Gribaudo, G.; Varani, S. Interplay between human cytomegalovirus and intrinsic/innate host responses: A complex bidirectional relationship. Mediators Inflamm. 2012, 2012, 607276. [CrossRef]

2. Britt, W. Manifestations of human cytomegalovirus infection: Proposed mechanisms of acute and chronic disease. Curr. Top. Microbiol. Immunol. 2008, 325, 417-470. 
3. Stern-Ginossar, N.; Weisburd, B.; Michalski, A.; Le, V.T.; Hein, M.Y.; Huang, S.X.; Ma, M.; Shen, B.; Qian, S.B.; Hengel, H.; et al. Decoding human cytomegalovirus. Science 2012, 338, 1088-1093. [CrossRef]

4. Cha, T.A.; Tom, E.; Kemble, G.W.; Duke, G.M.; Mocarski, E.S.; Spaete, R.R. Human cytomegalovirus clinical isolates carry at least 19 genes not found in laboratory strains. J. Virol. 1996, 70, 78-83. [CrossRef]

5. Murphy, E.; Yu, D.; Grimwood, J.; Schmutz, J.; Dickson, M.; Jarvis, M.A.; Hahn, G.; Nelson, J.A.; Myers, R.M.; Shenk, T.E. Coding potential of laboratory and clinical strains of human cytomegalovirus. Proc. Natl. Acad. Sci. USA 2003, 100, 14976-14981. [CrossRef]

6. Jenkins, C.; Abendroth, A.; Slobedman, B. A novel viral transcript with homology to human interleukin-10 is expressed during latent human cytomegalovirus infection. J. Virol. 2004, 78, 1440-1447. [CrossRef]

7. Dolan, A.; Cunningham, C.; Hector, R.D.; Hassan-Walker, A.F.; Lee, L.; Addison, C.; Dargan, D.J.; McGeoch, D.J.; Gatherer, D.; Emery, V.C.; et al. Genetic content of wild-type human cytomegalovirus. J. Gen. Virol. 2004, 85 Pt 5, 1301-1312. [CrossRef]

8. Umashankar, M.; Petrucelli, A.; Cicchini, L.; Caposio, P.; Kreklywich, C.N.; Rak, M.; Bughio, F.; Goldman, D.C.; Hamlin, K.L.; Nelson, J.A.; et al. A novel human cytomegalovirus locus modulates cell type-specific outcomes of infection. PLoS Pathog. 2011, 7, e1002444. [CrossRef]

9. Bughio, F.; Elliott, D.A.; Goodrum, F. An endothelial cell-specific requirement for the UL133-UL138 locus of human cytomegalovirus for efficient virus maturation. J. Virol. 2013, 87, 3062-3075. [CrossRef]

10. Li, G.; Rak, M.; Nguyen, C.C.; Umashankar, M.; Goodrum, F.D.; Kamil, J.P. An epistatic relationship between the viral protein kinase UL97 and the UL133-UL138 latency locus during the human cytomegalovirus lytic cycle. J. Virol. 2014, 88, 6047-6060. [CrossRef]

11. Grainger, L.; Cicchini, L.; Rak, M.; Petrucelli, A.; Fitzgerald, K.D.; Semler, B.L.; Goodrum, F. Stress-inducible alternative translation initiation of human cytomegalovirus latency protein pUL138. J. Virol. 2010, 84, 9472-9486. [CrossRef]

12. Le, V.T.; Trilling, M.; Hengel, H. The cytomegaloviral protein pUL138 acts as potentiator of tumor necrosis factor (TNF) receptor 1 surface density to enhance ULb'-encoded modulation of TNF-alpha signaling. J. Virol. 2011, 85, 13260-13270. [CrossRef]

13. Montag, C.; Wagner, J.A.; Gruska, I.; Vetter, B.; Wiebusch, L.; Hagemeier, C. The latency-associated UL138 gene product of human cytomegalovirus sensitizes cells to tumor necrosis factor alpha (TNF-alpha) signaling by upregulating TNF-alpha receptor 1 cell surface expression. J. Virol. 2011, 85, 11409-11421. [CrossRef]

14. Shenk, T.; Alwine, J.C. Human Cytomegalovirus: Coordinating Cellular Stress, Signaling, and Metabolic Pathways. Annu. Rev. Virol. 2014, 1, 355-374. [CrossRef]

15. Weekes, M.P.; Tomasec, P.; Huttlin, E.L.; Fielding, C.A.; Nusinow, D.; Stanton, R.J.; Wang, E.C.; Aicheler, R.; Murrell, I.; Wilkinson, G.W.; et al. Quantitative temporal viromics: An approach to investigate host-pathogen interaction. Cell 2014, 157, 1460-1472. [CrossRef]

16. Weekes, M.P.; Tan, S.Y.; Poole, E.; Talbot, S.; Antrobus, R.; Smith, D.L.; Montag, C.; Gygi, S.P.; Sinclair, J.H.; Lehner, P.J. Latency-associated degradation of the MRP1 drug transporter during latent human cytomegalovirus infection. Science 2013, 340, 199-202. [CrossRef]

17. Zhao, F.; Shen, Z.Z.; Liu, Z.Y.; Zeng, W.B.; Cheng, S.; Ma, Y.P.; Rayner, S.; Yang, B.; Qiao, G.H.; Jiang, H.F.; et al. Identification and BAC construction of Han, the first characterized HCMV clinical strain in China. J. Med. Virol. 2016, 88, 859-870. [CrossRef]

18. Preston, C.M.; Harman, A.N.; Nicholl, M.J. Activation of interferon response factor-3 in human cells infected with herpes simplex virus type 1 or human cytomegalovirus. J. Virol. 2001, 75, 8909-8916. [CrossRef]

19. Sivan, G.; Ormanoglu, P.; Buehler, E.C.; Martin, S.E.; Moss, B. Identification of Restriction Factors by Human Genome-Wide RNA Interference Screening of Viral Host Range Mutants Exemplified by Discovery of SAMD9 and WDR6 as Inhibitors of the Vaccinia Virus K1L-C7L-Mutant. MBio 2015, 6, e01122. [CrossRef]

20. Browne, E.P.; Wing, B.; Coleman, D.; Shenk, T. Altered cellular mRNA levels in human cytomegalovirus-infected fibroblasts: Viral block to the accumulation of antiviral mRNAs. J. Virol. 2001, 75, 12319-12330. [CrossRef]

21. Harwardt, T.; Lukas, S.; Zenger, M.; Reitberger, T.; Danzer, D.; Ubner, T.; Munday, D.C.; Nevels, M.; Paulus, C. Human Cytomegalovirus Immediate-Early 1 Protein Rewires Upstream STAT3 to Downstream STAT1 Signaling Switching an IL6-Type to an IFNgamma-Like Response. PLoS Pathog. 2016, 12, e1005748. [CrossRef] 
22. Petrucelli, A.; Rak, M.; Grainger, L.; Goodrum, F. Characterization of a novel Golgi apparatus-localized latency determinant encoded by human cytomegalovirus. J. Virol. 2009, 83, 5615-5629. [CrossRef]

23. Cusick, J.K.; Mustian, A.; Goldberg, K.; Reyland, M.E. RELT induces cellular death in HEK 293 epithelial cells. Cell. Immunol. 2010, 261, 1-8. [CrossRef]

24. Cohn, M.A.; Kowal, P.; Yang, K.; Haas, W.; Huang, T.T.; Gygi, S.P.; D'Andrea, A.D. A UAF1-containing multisubunit protein complex regulates the Fanconi anemia pathway. Mol. Cell 2007, 28, 786-797. [CrossRef]

25. Park, J.; Lee, B.S.; Choi, J.K.; Means, R.E.; Choe, J.; Jung, J.U. Herpesviral protein targets a cellular WD repeat endosomal protein to downregulate T lymphocyte receptor expression. Immunity 2002, 17, 221-233. [CrossRef]

26. Chai, F.; Li, H.Y.; Wang, W.; Zhu, X.J.; Li, Y.; Wang, S.; Guo, L.; Zhang, L.K.; Xiao, G. Subcellular quantitative proteomic analysis reveals host proteins involved in human cytomegalovirus infection. Biochim. et Biophys. Acta 2015, 1854, 967-978. [CrossRef]

27. Perez-Riverol, Y.; Csordas, A.; Bai, J.; Bernal-Llinares, M.; Hewapathirana, S.; Kundu, D.J.; Inuganti, A.; Griss, J.; Mayer, G.; Eisenacher, M.; et al. The PRIDE database and related tools and resources in 2019: Improving support for quantification data. Nucleic Acids Res. 2019, 47, D442-D450. [CrossRef]

28. Emmott, E.; Goodfellow, I. Identification of protein interaction partners in mammalian cells using SILAC-immunoprecipitation quantitative proteomics. J. Vis. Exp. 2014, 89, e51656. [CrossRef]

29. Zhang, L.K.; Lin, T.; Zhu, S.L.; Xianyu, L.Z.; Lu, S.Y. Global quantitative proteomic analysis of human glioma cells profiled host protein expression in response to enterovirus type 71 infection. Proteomics 2015, 15, 3784-3796. [CrossRef]

30. Huang da, W.; Sherman, B.T.; Lempicki, R.A. Systematic and integrative analysis of large gene lists using DAVID bioinformatics resources. Nat. Protoc. 2009, 4, 44-57. [CrossRef]

Sample Availability: Samples of pcDNA3.1-UL82 plasmid is available from the authors.

(C) 2020 by the authors. Licensee MDPI, Basel, Switzerland. This article is an open access article distributed under the terms and conditions of the Creative Commons Attribution (CC BY) license (http://creativecommons.org/licenses/by/4.0/). 